\title{
EXTREME NON-ARENS REGULARITY OF THE GROUP ALGEBRA
}

\author{
M. FILALI AND J. GALINDO
}

\begin{abstract}
The Banach algebras of Harmonic Analysis are usually far from being Arens regular and often turn out to be as irregular as possible. This utmost irregularity has been studied by means of two notions: strong Arens irregularity, in the sense of Dales and Lau, and extreme non-Arens regularity, in the sense of Granirer. Lau and Losert proved in 1988 that the convolution algebra $L^{1}(G)$ is strongly Arens irregular for any infinite locally compact group. In the present paper we prove that $L^{1}(G)$ is extremely non-Arens regular for any infinite locally compact group.

We actually prove the stronger result that for any non-discrete locally compact group $G$, there is a linear isometry from $L^{\infty}(G)$ into the quotient space $L^{\infty}(G) / \mathcal{F}(G)$, with $\mathcal{F}(G)$ being any closed subspace of $L^{\infty}(G)$ made of continuous bounded functions. This, together with the known fact that $\ell^{\infty}(G) / \mathcal{W} \mathcal{A P}(G)$ always contains a linearly isometric copy of $\ell^{\infty}(G)$, proves that $L^{1}(G)$ is extremely non-Arens regular for every infinite locally compact group.
\end{abstract}

\section{INTRODUCTION}

The second dual space $A^{* *}$ of a Banach algebra $A$ can be made into a Banach algebra with two different products, each extending the original product of $A$. These products were introduced by Arens in 1951 and are called the first (or left) Arens product and the second (or right) Arens product, see [1] and [2]. We may describe the Arens products explicitly as follows (although we shall have no need of that): If $\left(\mu_{\alpha}\right)$ and $\left(\nu_{\beta}\right)$ are nets in $A$ with $\lim _{\alpha} \mu_{\alpha}=\mu$ and $\lim _{\beta} \nu_{\beta}=\nu$, then

$$
\mu \circ \nu=\lim _{\alpha} \lim _{\beta} \mu_{\alpha} \nu_{\beta} \quad \text { and } \quad \mu \square \nu=\lim _{\beta} \lim _{\alpha} \mu_{\alpha} \nu_{\beta},
$$

where the limits are taken in the weak*-topology in $A^{* *}$ and the order of the limits is crucial. Note that $\mu \mapsto \mu \circ \nu$ is weak $^{*}$-weak ${ }^{*}$-continuous in $\mu$ for each fixed $\nu \in A^{* *}$, while $\nu \mapsto \mu \circ \nu$ is only weak ${ }^{*}$-weak ${ }^{*}$-continuous in $\nu$ for each fixed $\mu \in A$. It fails, in general, to be weak*-weak*- continuous in

Date: January 19, 2018.

2010 Mathematics Subject Classification. Primary 22D15; Secondary 43A46, 43A15, 43A60, 54H11.

Key words and phrases. group algebra, extremely non-Arens regular, weakly almost periodic, isometry, Haar homeomorphism, metrizable groups.

Research of the second named author supported by Universitat Jaume I, grant P1·B2014-35. 
$\nu$ when $\mu$ is not $A$. The topological centre of $A^{* *}$ is defined by

$$
\begin{aligned}
Z\left(A^{* *}\right) & =\left\{\mu \in A^{* *}: \nu \mapsto \mu \circ \nu \text { is weak }{ }^{*} \text {-weak }{ }^{*} \text {-continuous on } A^{* *}\right\} \\
& =\left\{\mu \in A^{* *}: \mu \circ \nu=\mu \square \nu \text { for all } \nu \in A^{* *}\right\} .
\end{aligned}
$$

As already noted, $A$ is a subalgebra of $Z\left(A^{* *}\right)$.

The same observations and definitions may be given for the second product with the roles of the variables reversed.

The algebra $A$ is said to be Arens regular if these two products coincide, which is the same as $Z\left(A^{* *}\right)=A^{* *}$. When $A$ is commutative, it is easy to check that $A$ is Arens regular if and only if $A^{* *}$ is commutative with respect to both products.

An interesting criterion for the Arens regularity of $A$ was given by Pym [45] in 1965 . He considered the space $\mathcal{W} \mathcal{A P}(A)$ of weakly almost periodic functionals on $A$, defined as the set of all $f \in A^{*}$ such that the linear map

$$
A \rightarrow A^{*}: a \mapsto f . a
$$

is weakly compact, where the action of $A$ on $A^{*}$ is given by

$$
<f . a, b>=<f, a b>, \quad a, b \in A, f \in A^{*} .
$$

These are the functionals $f \in A^{*}$ satisfying Grothendieck's double limit criterion

$$
\lim _{n} \lim _{m} f\left(a_{n} b_{m}\right)=\lim _{m} \lim _{n} f\left(a_{n} b_{m}\right)
$$

for any pair of sequences $\left(a_{n}\right)_{n},\left(b_{m}\right)_{m}$ in $A$ for which both limits exist. Pym proved that $A$ is Arens regular if and only if $\mathcal{W} \mathcal{A P}(A)=A^{*}$, see also [53]. For further details, see [18].

All $C^{*}$-algebras are Arens regular. This fact was first proved (implicitly) by Sherman [49] and Takeda [50] when they proved that the second conjugate of a $C^{*}$-algebra can be seen as an algebra of operators on a Hilbert space. Some years later, Civin and Yood [9, Theorem 7.1] reproduced Takeda's proof and brought up explicitly the Arens regularity of $C^{*}$-algebras. A different proof of this same fact can also be found in [5, Theorem 38.19]. For more details, see [10], [18] and [11].

The group algebra $L^{1}(G)$ of an infinite locally compact group, however, is never Arens regular. Arens himself showed that the semigroup algebra $\ell^{1}$ with convolution is non-Arens regular. To prove this fact he produced two distinct invariant means $\mu$ and $\nu$ in $\ell^{1^{* *}}$. It is then trivial to see that

$$
\nu \mu=\mu \neq \nu=\mu \nu,
$$

which means that $Z\left(\left(\ell^{1}\right)^{* *}\right) \neq\left(\ell^{1}\right)^{* *}$. In [13], Day used the same argument to show that $L^{1}(G)$ is non-Arens regular for many infinite discrete groups, including all Abelian ones. This same approach was followed in the seminal paper of Civin and Yood [9] where $L^{1}(G)$ was shown to be non-Arens regular for any infinite locally compact Abelian group. Their method relied again on Day's result when the group is discrete. When $G$ is not discrete, the Hahn Banach theorem provides a non-zero right annihilator $\mu$ of $L^{1}(G)^{* *}$, i.e., $L^{1}(G)^{* *} \mu=\{0\}$, and so

$$
\mu \nu=\mu \neq 0=\nu \mu \quad \text { for every right identity } \quad \nu \in L^{1}(G)^{* *} .
$$


The general case was finally settled by Young in 1973 in [54]. Young relied on the criterion proved in [45, Theorem 4.2] (see also [53, Theorem 10]) and showed how to produce, in each infinite locally compact group, a function in $L^{\infty}(G)$ that is not in $\mathcal{W} \mathcal{A} \mathcal{P}\left(L^{1}(G)\right)$.

Young's approach to non-Arens regularity of $L^{1}(G)$ was therefore different in essence from all previous ones. It passed through $\mathcal{W} \mathcal{A P}(A)$ while the others passed through the topological centre $Z\left(A^{* *}\right)$.

Depending on one's approach to non-Arens regularity (through $Z\left(A^{* *}\right)$ or through $\mathcal{W} \mathcal{A P}(A)$ ), two different ways to tell that a Banach algebra is as non-Arens regular as possible arise:

- According to Dales and Lau [11], the Banach algebra $A$ is said to be strongly Arens irregular, when $Z\left(A^{* *}\right)=A$.

- According to Granirer [25], $A$ is said to be extremely non-Arens regular $(E N A R)$ if the quotient space $A^{*} / \mathcal{W} \mathcal{A} \mathcal{P}(A)$ contains a closed linear subspace which has $A^{*}$ as a continuous linear image.

The group algebra is strongly Arens irregular for any infinite locally compact group. This was proved first by Isik, Pym and Ülger [37] when $G$ is compact, then by Grosser and Losert in [26] when $G$ is Abelian, and finally in the general case by Lau and Losert in [38]. A number of articles offering different approaches and various properties related to the topological centres appeared subsequently, we cite for example [11], [43], [17] and most recently [6].

Granirer himself proved in $[25]$ that the Fourier algebras $A(\mathbb{R})$ and $A(\mathbb{T})$, and therefore $L^{1}(\mathbb{R})$ and $\ell^{1}(\mathbb{Z})$, are extremely non-Arens regular. In [33], $\mathrm{Hu}$ generalized Granirer's results and proved that the Fourier algebra $A(G)$ is extremely non-Arens regular whenever $\chi(G) \geq \kappa(G)$, where $\kappa(G)$ is the minimal number of compact sets required to cover $G$ and $\chi(G)$ is the minimal cardinality of an open base at the identity $e$ of $G$.

In [23, Theorems 4.1 and 4.7], Fong and Neufang considered infinite, locally compact, metrizable groups with $\kappa(G) \geq \aleph_{0}$ and proved that the group algebra $L^{1}(G)$ is extremely non-Arens regular when $G$ is $\sigma$-compact and metrizable or it contains an open $\sigma$-compact, metrizable, subgroup $H$ which is either normal or has $|H|<|G|$.

In [19], Filali and Vedenjuoksu proved, using slowly oscillating functions, that the semigroup algebra $\ell^{1}(S)$ is extremely non-Arens regular for any infinite weakly cancellative discrete semigroup. In particular, this property is held by the group algebra $L^{1}(G)$ for any infinite discrete group $G$.

With a different approach, Bouziad and Filali [7] proved that the quotient space $\mathcal{L U} \mathcal{C}(G) / \mathcal{W} \mathcal{A P}(G)$ contains a copy of $\ell^{\infty}(\kappa(G))$ for every locally compact group $G$. Since $\mathcal{W} \mathcal{A P}(G)$ can be identified with $\mathcal{W} \mathcal{A P}\left(L^{1}(G)\right)$, this implies the full dual of Hu's result, i.e., that the group algebra $L^{1}(G)$ is extremely non-Arens regular whenever $\kappa(G) \geq \chi(G)$. The same conclusion was obtained again in [16], drawing upon results of [15], for non-discrete $G$, using the size of the quotient space $\mathcal{C B}(G) / \mathcal{L U} \mathcal{C}(G)$.

Here, $\mathcal{C} \mathcal{B}(G)$ is the space of all bounded, continuous, scalar-valued functions on $G$. The space $\mathcal{L U C}(G)$ consists of all bounded scalar-valued functions $f$ on $G$ are left norm continuous in the sense that

$$
s \mapsto{ }_{s} f: G \rightarrow \mathcal{C B}(G)
$$


is continuous. In the literature, these functions are also called right uniformly continuous since they are uniformly continuous with respect to the right uniformity of $G$, see for example [31]. The space $\mathcal{W} \mathcal{A P}(G)$ consists of all bounded, continuous, scalar-valued functions on $G$ which are weakly almost periodic, all with the supremum norm.

In this paper, we prove the full theorem.

Theorem A. The group algebra $L^{1}(G)$ is extremely non-Arens regular for any infinite locally compact group $G$.

As already noted, this result was proved in [7] and [19] when $G$ is discrete. So we are really concerned with the case when $G$ is not discrete. In this case, in fact, we shall prove the following stronger theorem.

Theorem B. Let $\mathcal{F}(G)$ be any closed subspace of $\mathcal{E} \mathcal{B}(G)$. Then there exists a linear isometric copy of $L^{\infty}(G)$ in the quotient space $L^{\infty}(G) / \mathcal{F}(G)$ for any non-discrete, locally compact group $G$.

As for the Fourier algebra $A(G)$, the situation is even more complex. The question of whether $A(G)$ is not Arens regular is still not completely settled for discrete non-amenable groups. When $G$ is an amenable locally compact group, the Fourier algebra $A(G)$ is Arens regular if and only if $G$ is finite. This was proved in 1989 by Lau and Wong, see [39]. One can deduce with Forrest [24] that $A(G)$ cannot be Arens regular if $G$ contains an infinite amenable subgroup. This implies for instance that $A\left(F_{2}\right)$, the Fourier algebra on the free group on two generators, is not Arens regular. A recent paper by Losert [41] shows that, unlike the group algebra $L^{1}\left(F_{2}\right)$, the Fourier algebra $A\left(F_{2}\right)$ is not strongly Arens irregular either. By applying [36, Corollary 5.6] to the argument in [24, Corollary 3.13] one also gets that $A(G)$ is never Arens regular when $G$ is nondiscrete.

Very little is known on extremely non-Arens regularity of the Fourier algebra. The only piece of information is, as already mentioned, in [33] where Hu proved that $A(G)$ is extremely non-Arens regular whenever $\chi(G) \geq \kappa(G)$. Our Theorem A enables us to omit this condition when $G$ is Abelian.

It may be worthwhile to note that extreme non-Arens regularity in the sense of Granirer does not imply strong Arens irregularity in the sense of [11] since $A(S U(3))$ is extremely non-Arens regular by [33], but it is not strongly Arens irregular as recently proved by Losert [40]. Neither does strong Arens irregularity imply extreme non-Arens regularity, see [35].

1.1. Outline of our approach. All the available proofs of extreme nonArens regularity of $L^{1}(G)$ proceed in two steps:

(i) $L^{\infty}(G)$ is embedded in $\ell^{\infty}(\mathfrak{m})$, where $\mathfrak{m}=\max \{\kappa(G), \chi(G)\}$ is the density character of $L^{1}(G)$, and

(ii) if $\mathfrak{n}=\max \left\{\kappa(G), \aleph_{0}\right\}$, a collection of $\mathfrak{n}$-many open disjoint subsets of $G$ with special properties is constructed and used to produce a copy of $\ell^{\infty}(\mathfrak{n})$ in the quotient space $L^{\infty}(G) / \mathcal{W} \mathcal{A P}(G)$.

This approach works when $\kappa(G) \geq \chi(G)$ or when $G$ is metrizable, but fails for large compact groups: one cannot hope for an uncountable collection of open disjoint subsets in a compact group $G$ (all compact groups have 
countable cellularity, see [3, Corollary 4.18]). Adding to this difficulty is the fact, proved by Rosenthal [48, Proposition 4.7, Theorem 4.8], that for $\kappa>\aleph_{0}, \ell^{\infty}\left(\aleph_{0}\right)$ does not embed in $L^{\infty}(G)$, for any compact group $G$ (and hence there is little hope to find such an embedding in the quotient space $L^{\infty}(G) / \mathcal{W} \mathcal{A P}(G)$ either) regardless of the size of $\chi(G)$.

Let $w(G)$ be the weight of the group $G$, i.e., the the smallest cardinal number $m$ such that $G$ has a base of cardinality $m$. Recall that for every compact group with an uncountable $w(G)$, Theorem 3.1 of [27] together with Maharam's theorem (see, e.g., [22, Theorem 331I]) shows that $L^{1}(G)$ and $L^{1}\left(\{0,1\}^{w(G)}\right)$ are isometrically isomorphic as Banach spaces, and that $L^{\infty}(G)$ and $L^{\infty}\left(\{0,1\}^{w(G)}\right)$ are isomorphic as $\mathrm{W}^{*}$-algebras. Notwithstanding the strength of Maharam's theorem, the problem of extreme non-Arens regularity of $L^{1}(G)$ for $G$ compact cannot be reduced to that of $L^{1}\left(\{0,1\}^{w(G)}\right)$ since the multiplicative structure of the Banach algebra has to be taken into account. A dramatic illustration of this is easily available for non-compact groups: $\ell^{1}(\mathbb{Z})$ is an extremely non-Arens regular algebra but, if its multiplication (i.e. convolution), is replaced by pointwise multiplication, the resulting Banach algebra is Arens-regular, [44, 1.4.8 and 1.4.9].

We need therefore an alternative approach to reach our aim. Our strategy shall rely on the following three keys:

- We first deal with groups of the form $G=M \times H$, with $M$ a nondiscrete, metrizable locally compact group. For such a group $G$, we show in Theorem 3.1 that the quotient $L^{\infty}(G) / \mathcal{C B}(G)$ contains a copy of $\ell^{\infty}\left(L^{\infty}(H)\right)$, a result inspired by [16, Theorem 2.11] whose ideas date back at least to [8]. This theorem is already used in Corollary 3.3 to deduce that $L^{1}(G)$ is extremely non-Arens regular for groups of this form if $M$ is, in addition, $\sigma$-compact. But this leaves out in particular the case when $H=\{e\}$ and $M$ is a compact non-metrizable group, i.e., when our group $G$ is compact and nonmetrizable. Our second main tool takes care of this case.

- The second key is Lemma 4.2 that isolates a set of conditions that, as a consequence of the work of Grekas and Mercourakis [28], are known to be satisfied by compact groups. These conditions in particular imply that every compact group $K$ has a quotient topologically isomorphic to a product of $w(K)$-many metrizable factors. This, together with our first key, will lead to Theorem 5.4 to the effect that the quotient space $L^{\infty}(K) / \mathcal{C B}(K)$ contains a linear isometric copy of $\left.L^{\infty}(K)\right)$ for any infinite compact group. Thus, $L^{1}(K)$ is extremely non-Arens regular for any infinite compact group $K$.

- The final step will be based on a theorem of Davis [12], reminiscent of results of Yamabe [52]. We see that the problem of finding a linear isometric copy of $L^{\infty}(G)$ in $L^{\infty}(G) / \mathcal{C B}(G)$ can be reduced to the problem of finding a special sort of isometry between $L^{\infty}\left(G_{0}\right)$ and $L^{\infty}\left(G_{0}\right) / \mathcal{C B}\left(G_{0}\right)$ for some open subgroup $G_{0}$ of $G$. The extreme non-Arens regularity of the group algebra of any locally compact group is then reduced to that of $L^{1}\left(\mathbb{R}^{n} \times K\right)$, where $K$ is a compact group. Corollary 3.3 and Corollary 5.4 lead then immediately to the proof of Theorem B. Since we know from [7] or [19] that 
the group algebra is extremely non-Arens regular for any infinite discrete group, Theorem $\mathrm{A}$ is then an immediate consequence.

\section{Notation AND TERMinOlOGY}

Being concerned as we are with isometries into quotients of $L^{\infty}(G)$-spaces we deal with these spaces and their subspaces, with locally compact groups and their Haar measures and with Banach spaces and isometries between them. We summarize here our terminology on these subjects.

2.1. Cardinal invariants, topological groups and Haar measure. We will be considering several cardinal invariants of a topological space $X$.

The density character of $X, d(X)$ is the least cardinality of a dense subset of $X$. The local weight $\chi(X)$ of $X$ is the least cardinality of an open base at the identity of $X$. The topological weight $w(X)$ of $X$ is the least cardinality of an open base of $X$. The compact covering number $\kappa(X)$ of $X$ is the least cardinality of a compact covering of $X$. We recall that for an infinite compact group $G, w(G)=\chi(G)$.

If $G$ is a locally compact group, then $\lambda_{G}$ will denote the left Haar measure of $G$. The characteristic function of a set $V$ will be denoted by $\chi_{V}$.

2.2. Subspaces of $L^{\infty}(G)$. We recall that $L^{1}(G)$ is the Banach space made of all equivalence classes of scalar-valued functions which are integrable with respect to the Haar measure $\lambda_{G}$, where as usual, two functions are equivalent if and only if they differ only on a set of Haar measure zero. By $L^{\infty}(G)$ we understand the Banach dual of $L^{1}(G)$.

Our realization of $L^{\infty}(G)$, valid for a general locally compact group, requires the concept of locally null set. A subset $A \subset G$ is locally null if $A \cap K$ is of Haar measure zero for every compact set $K \subset G$ and two functions $f, g: G \rightarrow \mathbb{C}$ are equal locally almost everywhere (l.a.e.), if there is a locally null subset $A$ such that $f(x)=g(x)$ for all $x \notin A$. Following [31, Section 12], [20, Page 46] or [21, Exercises 6.23-26] (and departing from the most usual definition), we identify $L^{\infty}(G)$ with the vector space of all equivalence classes of essentially bounded and locally measurable functions. Two functions are equivalent if and only if they are equal locally almost everywhere. The norm is then given by

$$
\|f\|_{L^{\infty}(G)}=\inf \{c:|f(x)| \leq c \text { l.a.e. }\} .
$$

Note that (see, for instance, the proof of [31, Theorem 12.18])

$$
\|f\|_{L^{\infty}(G)}=\sup \left\{\left\|f \chi_{K}\right\|: K \subset G \text { is compact }\right\} .
$$

When there is no confusion, we simply write $\|f\|$ for the norm of $f$ in $L^{\infty}(G)$. If $G$ is $\sigma$-compact (so that Haar measure is $\sigma$-finite), a subset $A \subset G$ is null if and only if it is locally null, hence this construction of $L^{\infty}(G)$ coincides with the standard one as $L^{\infty}\left(G, \lambda_{G}\right)$.

Readers who prefer to define $L^{\infty}(G)$ in the standard way with the functions being identified when they are equal almost everywhere are directed for example to Royden in [47]. Royden uses Radon measures in the sense of Schwartz and inner regularity rather than outer regularity, as in [31] or [20].

The space $\mathcal{C} \mathcal{B}(G)$ will stand for the Banach subspace of $L^{\infty}(G)$ consisting on all continuous and bounded functions on $G$. In this paper the 
subspace $\mathcal{W} \mathcal{A P}(G)$ of $\mathcal{C B}(G)$ will play a special role. A function $f \in \mathcal{C} \mathcal{B}(G)$ is in $\mathcal{W} \mathcal{A P}(G)$, i.e., is weakly almost periodic when the set of its left (or equivalently, right) translates $\left\{f_{s}: s \in G\right\}$ is relatively weakly compact in $\mathrm{e} \mathcal{B}(G)$. Grothendieck's famous iterated limit criterion shows that a function $f \in \mathcal{C B}(G)$ is in $\mathcal{W} \mathcal{A P}(G)$ if and only if, for any sequences $\left(x_{n}\right)$ and $\left(y_{m}\right)$ in $G$,

$$
\lim _{n \rightarrow \infty} \lim _{m \rightarrow \infty} f\left(x_{n} y_{m}\right)=\lim _{m \rightarrow \infty} \lim _{n \rightarrow \infty} f\left(x_{n} y_{m}\right)
$$

whenever these iterated limits exist; see for example [4, Appendix A].

As indicated in the introduction, $\mathcal{W} \mathcal{A P}(A)$ is the space of all weakly almost periodic functionals of a Banach algebra $A$. Ülger [51] proved that $\mathcal{W} \mathcal{A} \mathcal{P}\left(L^{1}(G)\right)$ equals $\mathcal{W} \mathcal{A P}(G)$ for every locally compact group $G$.

2.3. Banach spaces and isometries. If $E$ is a Banach space and $I$ is a nonempty index set, $\ell^{\infty}(I, E)$ will denote as usual the linear space of all families $\mathbf{x}=\left(x_{i}\right)_{i \in I}$ with $x_{i} \in E$ and with $\sup \left\{\left\|x_{i}\right\|_{E}: i \in I\right\}<\infty$. Equipped with the norm

$$
\|\mathbf{x}\|=\sup \left\{\left\|x_{i}\right\|_{E}: i \in I\right\},
$$

$\ell^{\infty}(I, E)$ turns into a Banach space. The particular case $\ell^{\infty}(\mathbb{N}, E)$ will be be denoted simply as $\ell^{\infty}(E)$. Of course, when $|I|=1, \ell^{\infty}(I, E)$ is just the Banach space $E$. When $E=\mathbb{C}, \ell^{\infty}(I, E)$ will be denoted as $\ell^{\infty}(\eta)$ with $\eta=|I|$.

By an isometry between two Banach spaces $E_{1}$ and $E_{2}$ we understand a map $T_{1}: E_{1} \rightarrow E_{2}$ with $\|T(v)\|_{E_{2}}=\|v\|_{E_{1}}$ for all $v \in E_{1}$, i.e. we do not assume that isometries are onto.

We record here an elementary fact, and the concept it relates to, that will be often used to find isometries into quotients.

Definition 2.1. Let $T: \mathcal{E} \rightarrow \mathcal{F}$ be a linear isometry between Banach spaces. If $\mathcal{G}$ is a closed subspace of $\mathcal{F}$, we say that $T$ is preserved by $\mathcal{G}$ when the following property holds:

$$
\|T \xi-\phi\| \geq\|\xi\|, \quad \text { for all } \phi \in \mathcal{G} \text { and } \xi \in \mathcal{E} .
$$

Lemma 2.2. Let $T: \mathcal{E} \rightarrow \mathcal{F}$ be an isometry between Banach spaces and let $\mathcal{H}, \mathcal{G}$ be closed linear subspaces of $\mathcal{F}$ with $\mathcal{H} \subseteq \mathcal{G}$. Denote by $Q: \mathcal{F} \rightarrow \mathcal{F} / \mathcal{H}$ the quotient map. If $T$ is preserved by $\mathcal{G}$, then the map $Q \circ T: \mathcal{E} \rightarrow \mathcal{F} / \mathcal{H}$ is a linear isometry.

Proof. Let $\xi \in \mathcal{E}$. We first observe that $T$ is also preserved by $\mathcal{H}$ and hence:

$$
\|Q(T(\xi))\|_{\mathcal{F} / \mathcal{H}}=\inf \left\{\|T(x)+\phi\|_{\mathcal{F}}: \phi \in \mathcal{H}\right\} \geq\|\xi\|_{\mathcal{E}} .
$$

Since $T$ is an isometry, the converse inequality also follows:

$$
\|Q(T(\xi))\|_{\mathcal{F} / \mathcal{H}} \leq\|T(\xi)\|_{\mathcal{F}}=\|\xi\|_{\mathcal{E}} .
$$

Next we show how extreme non-Arens regularity of a locally compact group can be lifted from open subgroups. 


\section{Groups with a metrizable factor}

In this Section we deal with locally compact groups of the form $G=M \times$ $H$, with $M$ metrizable and $H$ any locally compact group. Two key results are proved. In our first key theorem, metrizability and non-discreteness of the factor group $M$ are used to get a copy of $\ell^{\infty}\left(L^{\infty}(H)\right)$ in the quotient space $L^{\infty}(M \times H) / \mathcal{C B}(M \times H)$. The second result shows in particular that $L^{\infty}(M \times H)$ is contained in $\ell^{\infty}\left(L^{\infty}(H)\right)$ when $M$ is also $\sigma$-compact. Non-discreteness is not necessary for the second result.

3.1. $L^{\infty}(M \times H) / \mathcal{C B}(M \times H)$ contains $\ell^{\infty}\left(L^{\infty}(H)\right)$. For our first key result, both metrizability and non-discreteness of $M$ are needed. Our argument is reminiscent of [16, Lemma 2.7]. For trivial $H$ similar results were obtained in [30, Lemma 5.2] and [7, Theorem 4.5].

Theorem 3.1. Let $H$ be a locally compact group and let $M$ be a non-discrete metrizable locally compact group. Then there exists a linear isometry

$$
\Psi: \ell^{\infty}\left(L^{\infty}(H)\right) \rightarrow L^{\infty}(M \times H)
$$

that is preserved by $\mathcal{C B}(M \times H)$.

Proof. Let $\left\{U_{n}\right\}_{n \in \mathbb{N}}$ denote a basis of neighborhoods of the identity in $M$ with $U_{n+1}^{2} \subset U_{n}$ for every $n \in \mathbb{N}$. Put, for each $n \in \mathbb{N}, V_{n}=U_{n} \backslash \overline{U_{n+1}}$, and consider the disjoint family of open sets in $M$ given by $\left\{V_{n}: n \in \mathbb{N}\right\}$. Next, partition $\mathbb{N}$ into infinitely many infinite subsets $I_{m}$. Enumerate each $I_{m}$ as $I_{m}=\left\{m_{n}: n \in \mathbb{N}\right\}$, with $m_{n}<m_{n+1}$.

For each $m \in \mathbb{N}$, define $f_{m}: M \times H \rightarrow \mathbb{C}$ as

$$
f_{m}(s, t)=\left\{\begin{array}{l}
1, \quad \text { if } s \in V_{m_{2 n}}, \\
-1, \quad \text { if } s \in V_{m_{2 n+1}}, \\
0, \quad \text { otherwise. }
\end{array}\right.
$$

Then $f_{m} \in L^{\infty}(M \times H)$, note that $f_{m}$ is simply

$$
f_{m}=\chi_{\left(\cup_{n} V_{m_{2 n}}\right) \times H}-\chi_{\left(\cup_{n} V_{m_{2 n+1}}\right) \times H} .
$$

Define now for each $\xi=\left(\xi_{m}\right)_{m \in \mathbb{N}} \in \ell^{\infty}\left(L^{\infty}(H)\right)$, the function $f_{\xi} \in$ $L^{\infty}(M \times H)$ by

$$
f_{\xi}(s, t)=\sum_{m \in \mathbb{N}} \xi_{m}(t) f_{m}(s, t) .
$$

Observe that $f_{\xi}$ is well-defined; in fact depending on where the variable $s$ is, $f_{\xi}(s, t)=\xi_{m}(t),-\xi_{m}(t)$ or 0 .

Then, consider the map $\Psi: \ell^{\infty}\left(L^{\infty}(H)\right) \rightarrow L^{\infty}(M \times H)$ given by

$$
\Psi(\xi)=f_{\xi} .
$$

It is easily observed that $\Psi$ is linear and $\|\Psi(\xi)\|=\left\|f_{\xi}\right\| \leq\|\xi\|$ for every $\xi=\left(\xi_{n}\right)_{n \in \mathbb{N}} \in \ell^{\infty}\left(L^{\infty}(H)\right)$.

Moreover, since for each $s \in V_{m_{2 n}}, f_{\xi}(s, t)=\xi_{m}(t)$, we deduce that

$$
\|\Psi(\xi)\|=\left\|f_{\xi}\right\|=\|\xi\| .
$$

We now prove that $\Psi$ is preserved by $\mathcal{C B}(M \times H)$. Since $\Psi$ is linear, it will be enough to show that

$\left\|f_{\xi}-\phi\right\| \geq 1$ for every $\xi \in \ell^{\infty}\left(L^{\infty}(H)\right)$ with $\|\xi\|=1$ and $\phi \in \mathcal{C B}(M \times H)$. 
Suppose, otherwise, that for some $\varepsilon>0$ and some $\phi \in \mathcal{C} \mathcal{B}(M \times H)$, we have

$$
\left\|f_{\xi}-\phi\right\|<1-\varepsilon
$$

Since $\|\xi\|=\sup \left\{\left\|\xi_{m}\right\|: m \in \mathbb{N}\right\}=1$, and for each $m$,

$$
\left\|\xi_{m}\right\|=\sup \left\{\left\|\xi_{m} \chi_{K}\right\|: K \subset H \text { is compact }\right\},
$$

we may fix $m \in \mathbb{N}$ and $K \subset H$ such that

$$
\left\|\xi_{m} \chi_{K}\right\|>1-\varepsilon / 2 \text {. }
$$

By (1), the set

$$
\mathcal{D}=\left\{(s, t) \in U_{0} \times K:\left|f_{\xi}(s, t)-\phi(s, t)\right| \geq 1-\varepsilon\right\}
$$

is the intersection of a locally null set and a compact set, it is therefore null. By Fubini's theorem (Fubini's theorem is applicable since we are effectively working on a compact set), there is a null set $A \subset M$ such that $s \in M \backslash A$ implies that the set

$$
\mathcal{C}_{s}=\{t \in H:(s, t) \in \mathcal{D}\}
$$

is null. The set $A$ can actually be described as

$$
A=\left\{s \in G: \lambda_{H}\left(\mathcal{C}_{s}\right)>0\right\} .
$$

Let now $n \in \mathbb{N}$ be picked arbitrarily.

Being null, $A$ cannot contain either of the open sets $V_{m_{2 n}}$ or $V_{m_{2 n+1}}$. We then choose

$$
s_{m_{2 n}} \in V_{m_{2 n}} \backslash A \text { and } s_{m_{2 n+1}} \in V_{m_{2 n+1}} \backslash A .
$$

Since $f_{m}$ takes the value 1 on $V_{m_{2 n}} \times H$ and the value -1 on $V_{m_{2 n+1}} \times H$, we see that, for every $t \in K \backslash\left(\mathcal{C}_{s_{m_{2 n}}} \cup \mathcal{C}_{s_{m_{2 n+1}}}\right)$ :

$$
\begin{aligned}
\left|\xi_{m}(t)-\phi\left(s_{m_{2 n}}, t\right)\right| & <1-\varepsilon, \text { and } \\
\left|\xi_{m}(t)+\phi\left(s_{m_{2 n+1}}, t\right)\right| & <1-\varepsilon .
\end{aligned}
$$

On the other hand, by our assumption (2), we may pick $t \in K \backslash \bigcup_{k} \mathcal{C}_{s_{m_{k}}}$ such that

$$
\left|\xi_{m}(t)\right| \geq 1-\frac{\varepsilon}{2}
$$

and we may suppose that $\xi_{m}(t)>0$.

Then, by (3), (4) and (5), we have for every $n \in \mathbb{N}$,

$\left|\phi\left(s_{m_{2 n}}, t\right)-\phi\left(s_{m_{2 n+1}}, t\right)\right| \geq\left|2 \xi_{m}(t)\right|-\left|\xi_{m}(t)-\phi\left(s_{m_{2 n}}, t\right)\right|-\left|\xi_{m}(t)+\phi\left(s_{m_{2 n+1}}, t\right)\right| \geq \varepsilon$.

Since, by construction, $\left(s_{m_{n}}\right)$ converges to $e$, this inequality goes against the continuity of $\phi$ at $(e, t)$.

3.2. $\mathbf{L}^{\infty}(\mathbf{M} \times \mathbf{H})$ is contained in $\ell^{\infty}\left(\mathbf{L}^{\infty}(\mathbf{H})\right)$. For our second main lemma, we recall that, for any given locally compact group, $d\left(L^{p}(G)\right) \leq \max \{\kappa(G), \chi(G)\}$, if $1 \leq p<\infty$, see [34, Lemma 7.5]. In particular, $d\left(L^{1}(G)\right)=\aleph_{0}$ when $G$ is an infinite, $\sigma$-compact, metrizable locally compact group.

Lemma 3.2. Let $M$ and $H$ be locally compact groups. There is then a linear isometry of $L^{\infty}(M \times H)$ into $\ell^{\infty}\left(I, L^{\infty}(H)\right)$ with $|I|=d\left(L^{1}(M)\right)$. 
Proof. Let $\left\{u_{i}: i \in I\right\}$ be a norm dense subset in the unit ball of $L^{1}(M)$. Let $\lambda_{M}$ and $\lambda_{H}$ be fixed left Haar measures on $M$ and $H$, respectively. We define a map:

$$
\begin{aligned}
\Psi: L^{\infty}(M \times H) & \rightarrow \ell^{\infty}\left(I, L^{\infty}(H)\right) \\
f & \mapsto\left(\Psi(f)_{i}\right)_{i \in I},
\end{aligned}
$$

where $\Psi(f)_{i}$ is given for every $v \in L^{1}(H)$ by

$$
<\Psi(f)_{i}, v>=\int_{M}\left(\int_{H} f(s, t) u_{i}(s) v(t) d \lambda_{H}(t)\right) d \lambda_{M}(s) .
$$

We claim than $\Psi$ is a linear isometry of $L^{\infty}(M \times H)$ into $\ell^{\infty}\left(I, L^{\infty}(H)\right)$.

$\Psi(f)_{i}$ defines obviously a linear functional on $L^{1}(H)$ for each $i<d$. In addition,

(6)

$$
\begin{aligned}
\left|<\Psi(f)_{i}, v>\right| & \leq \int_{M}\left(\int_{H}|f(s, t)| \cdot\left|u_{i}(s)\right| \cdot|v(t)| d \lambda_{H}(t)\right) d \lambda_{M}(s) \\
& \leq\|f\|_{L^{\infty}(M \times H)} \cdot\left(\int_{M}\left|u_{i}(s)\right| d \lambda_{M}(s)\right) \cdot\left(\int_{H}|v(t)| d \lambda_{H}(t)\right) \\
& \leq\|f\|_{L^{\infty}(M \times H)}\|v\|_{L^{1}(H)} .
\end{aligned}
$$

Being a continuous linear functional on $L^{1}(H), \Psi(f)_{i}$ can indeed be identified with an element of $L^{\infty}(H)$.

Inequality (6) actually gives:

$$
\|\Psi(f)\|_{\ell^{\infty}\left(d, L^{\infty}(H)\right)} \leq\|f\|_{L^{\infty}(M \times H)} .
$$

We now check the converse inequality. For every $i \in I$ and every function $v$ in the unit ball of $L^{1}(H)$, we have

$$
\begin{aligned}
\|\Psi(f)\|_{\ell^{\infty}\left(I, L^{\infty}(H)\right)} & =\sup _{j \in I}\left\|\Psi(f)_{j}\right\|_{L^{\infty}(H)} \\
& =\sup _{j \in I} \sup \left\{\left|<\Psi(f)_{j}, w>\right|:\|w\|_{L^{1}(H)} \leq 1\right\} \\
& \geq\left|<\Psi(f)_{i}, v>\right| \\
& =\left|\int_{M}\left(\int_{H} f(s, t) u_{i}(s) v(t) d \lambda_{H}(t)\right) d \lambda_{M}(s)\right| .
\end{aligned}
$$

Accordingly,

$$
\|\Psi(f)\|_{\ell^{\infty}\left(d, L^{\infty}(H)\right)} \geq\left|\int_{M}\left(\int_{H} f(s, t) u(s) v(t) d \lambda_{H}(t)\right) d \lambda_{M}(s)\right|
$$

for every $u$ and $v$ in the unit balls of $L^{1}(M)$ and $L^{1}(H)$, respectively.

Now, the set of maps

$\left\{(s, t) \mapsto u(s) v(t): u \in L^{1}(M)\right.$ and $v \in L^{1}(H)$ with $\left.\|u\|_{L^{1}(M)} \leq 1,\|v\|_{L^{1}(H)} \leq 1\right\}$

is dense in the unit ball of $L^{1}(M \times H)$ (it contains the characteristic function of every measurable rectangle of integrable sides). Therefore,

$$
\|\Psi(f)\|_{\ell^{\infty}\left(d, L^{\infty}(H)\right)} \geq\left|\int_{M}\left(\int_{H} f(s, t) h(s, t) d \lambda_{H}(t)\right) d \lambda_{M}(s)\right|
$$


for every $h$ in the unit ball of $L^{1}(M \times H)$. In other words,

$$
\|\Psi(f)\|_{\ell^{\infty}\left(d, L^{\infty}(H)\right)} \geq\|f\|_{L^{\infty}(M \times H)},
$$

as required.

We can already give the first consequence on the extreme non-Arens regularity of the group algebra for some locally compact groups.

Corollary 3.3. Let $G=H \times M$ be a locally compact group with $M$ nondiscrete, $\sigma$-compact and metrizable. Then there exists a linear isometry from $L^{\infty}(G)$ into $L^{\infty}(G)$ which is preserved by $\mathcal{C B}(G)$. In particular, the group algebra $L^{1}(G)$ is extremely non-Arens regular.

Proof. Let $\Psi_{1}$ denote the isometry preserved by $\mathcal{C B}(M \times H)$ constructed in Lemma 3.1 and denote by $\Psi_{2}$ the isometry obtained in Lemma 3.2 for $d=d\left(L^{1}(M)\right)=\aleph_{0}$,

$$
L^{\infty}(M \times H) \stackrel{\Psi_{2}}{\longrightarrow} \ell^{\infty}\left(L^{\infty}(H)\right) \stackrel{\Psi_{1}}{\longrightarrow} L^{\infty}(M \times H) .
$$

The composition $T=\Psi_{1} \circ \Psi_{2}$, is then an isometry preserved by $\mathcal{C} \mathcal{B}(M \times H)$ :

$$
\|T \xi-\phi\|=\left\|\Psi_{1}\left(\Psi_{2} \xi\right)-\phi\right\| \geq\left\|\Psi_{2} \xi\right\|=\|\xi\| .
$$

Since $\mathcal{W} \mathcal{A P}(G) \subset \mathcal{C B}(M \times H)$, Lemma 2.2, applied to $\mathcal{E}=\mathcal{F}=L^{\infty}(G)$ and $\mathcal{H}=\mathcal{W} \mathcal{A P}(G)$, proves there is a linear isometry of $L^{\infty}(G)$ into $L^{\infty}(G) / \mathcal{W} \mathcal{A P}(G)$. The group algebra $L^{1}(G)$ is therefore extremely non-Arens regular.

\section{ONE MORE LEMMA}

Our second key lemma in this section isolates a set of conditions which suffice to guarantee a linear isometric copy of $L^{\infty}(G)$ in the quotient space $L^{\infty}(G) / \mathcal{C B}(G)$, and so the extreme non-Arens regularity of the group algebra. It will be the subject of our next section to observe that these conditions are satisfied by compact groups.

We begin with a lemma that brings out a tool usually related to Weil formula, essential in the construction of Haar measure on quotients. Our arguments here draw upon Section 20 of [31], Section 3.4 of [46] and Section 2.6 of $[20]$.

Lemma 4.1. Let $G$ and $H$ be two locally compact groups and let $\varphi: G \rightarrow H$ be a continuous and open surjective homomorphism with compact kernel $N$. Then every $\psi \in L^{\infty}(G)$ induces a function $T_{N} \psi \in L^{\infty}(H)$ with the following properties:

(i) $\left\|T_{N} \psi\right\| \leq\|\psi\|$.

(ii) $T_{N}(\xi \circ \varphi)=\xi$ for every $\xi \in L^{\infty}(H)$.

(iii) If $\psi \in \mathcal{C B}(G)$, then $T_{N} \psi \in \mathcal{C} \mathcal{B}(H)$.

Proof. Let $\lambda_{N}$ be the normalized Haar measure on $N$ and consider, for each $\psi \in L^{\infty}(G)$, the convolution $\psi * \lambda_{N} \in L^{\infty}(G)$ :

$$
\left(\psi * \lambda_{N}\right)=\int_{N} \psi(x n) d \lambda_{N}(n) \quad \text { for } \quad x \in G
$$

By [31, Theorem 20.13], $\left\|\psi * \lambda_{N}\right\| \leq\|\psi\|$. 
Due to the invariance of $\lambda_{N}$ on $N,\left(\psi * \lambda_{N}\right)$ is constant on each coset of $N$. It therefore induces a function $T_{N} \psi$ on $H$ such that

$$
T_{N} \psi \circ \varphi=\left(\psi * \lambda_{N}\right) \text {. }
$$

We now check that this function is in $L^{\infty}(H)$. Let to that end $c>0$ be chosen such that

$$
\left\{x \in G:\left|\left(\psi * \lambda_{N}\right)(x)\right|>c\right\}
$$

is locally null. We claim that the set

$$
\left\{y \in H:\left|T_{N} \psi(y)\right|>c\right\}
$$

is also locally null. To see this, let $A$ be any compact subset of $H$. Then $\varphi^{-1}(A)$ is compact as well (continuous homomorphisms with compact kernel are proper maps) and, by assumption, the set

$$
\varphi^{-1}\left(\left\{\varphi(x) \in A:\left|\left(\psi * \lambda_{N}\right)(x)\right|>c\right\}\right)=\left\{x \in \varphi^{-1}(A):\left|\left(\psi * \lambda_{N}\right)(x)\right|>c\right\}
$$

is null. Using [20, Theorem 2.64] and writing each $y \in H$ as $\varphi(x)$ for some $x \in G$, we deduce that the set

$$
\left\{y \in A:\left|T_{N} \psi(y)\right|>c\right\}=\left\{\varphi(x) \in A:\left|\psi * \lambda_{N}\right|>c\right\},
$$

is also null. Therefore, $\left\|T_{N} \psi\right\| \leq\left\|\left(\psi * \lambda_{N}\right)\right\| \leq\|\psi\|$, as required.

Since $\varphi$ is a homomorphism, an straightforward computation that $T_{N}(\xi \circ$ $\varphi)=\xi$ for every $\xi \in L^{\infty}(H)$.

To prove Statement (iii), we consider $\psi \in \mathcal{C} \mathcal{B}(G)$. If $K$ is a compact subset of $G,\left.\left(\psi * \lambda_{N}\right)\right|_{K}$ is easily seen to be continuous as a consequence of $\left.\psi\right|_{K^{2}}$ being uniformly continuous. This implies that $\left(\psi * \lambda_{N}\right)$ is continuous. Since $\varphi$ is a quotient map, the continuity of $T_{N} \psi$ follows.

Here is now our second key lemma. The mapping $\varphi$ of Lemma 4.2, that will be explicitly produced in Theorem 5.4, induce the rest of the isometries.

Lemma 4.2. Let $K$ be a locally compact group and suppose that two locally compact groups $H$ and $M$ can be found such that:

(i) $M$ is metrizable, $\sigma$-compact and non-discrete,

(ii) there exists a linear isometry $\boldsymbol{\Psi}_{1}: L^{\infty}(K) \rightarrow L^{\infty}(M \times H)$,

(iii) there exists a continuous and open surjective homomorphism with compact kernel $K \stackrel{\varphi}{\longrightarrow} M \times H$.

Then there exists a linear isometry of $L^{\infty}(K)$ into $L^{\infty}(K)$ that is preserved by $\mathcal{C B}(K)$.

Proof. The desired isometry will be obtained as the composition of four isometries, the first of them being the one hypothesized in (ii).

The second and third isometries are obtained from Lemma 3.2 and Theorem 3.1 as in Corollary 3.3.

$$
\begin{aligned}
& \Psi_{2}: L^{\infty}(M \times H) \rightarrow \ell^{\infty}\left(L^{\infty}(H)\right) \\
& \Psi_{3}: \ell^{\infty}\left(L^{\infty}(H)\right) \longrightarrow L^{\infty}(M \times H) .
\end{aligned}
$$

Recall that $\boldsymbol{\Psi}_{3}$ is preserved by $\mathcal{C B}(M \times H)$.

The map $\varphi$ induces the fourth linear isometry:

$$
\boldsymbol{\Psi}_{4}: L^{\infty}(M \times H) \longrightarrow L^{\infty}(K)
$$


in the obvious way: $\boldsymbol{\Psi}_{4}(\xi)=\xi \circ \varphi$.

Putting everything together, we obtain the following chain of isometries:

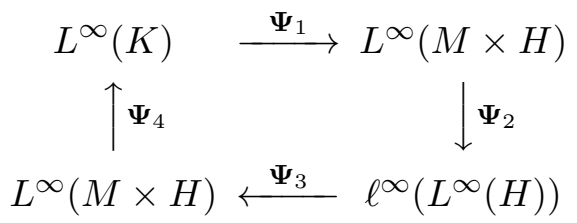

We finally check that the composition of all four isometries is preserved by $\mathcal{C} \mathcal{B}(K)$. It will be enough to check that $\Psi_{4} \circ \Psi_{3}$ is preserved by $\mathcal{C} \mathcal{B}(K)$.

Let to that end $\xi \in \ell^{\infty}\left(L^{\infty}(H)\right)$ and $\phi \in \mathcal{C B}(K)$. Let $T_{N} \phi \in \mathcal{C B}(M \times H)$ be the function provided in Lemma 4.1 for $\phi$ and $\varphi$. Using the properties deduced in that Lemma and that $\Psi_{3}$ is preserved by $\mathcal{C B}(K)$ we have that, for every $\xi \in \ell^{\infty}\left(L^{\infty}(H)\right)$,

$$
\left\|\Psi_{4}\left(\Psi_{3}(\xi)\right)-\phi\right\| \geq\left\|T_{N}\left(\Psi_{4}\left(\Psi_{3}(\xi)\right)-\phi\right)\right\|=\left\|\Psi_{3}(\xi)-T_{N} \phi\right\| \geq\|\xi\| .
$$

The isometry $\Psi_{4} \circ \Psi_{3}$ is therefore preserved by $\mathcal{C} \mathcal{B}(K)$.

\section{The COMPaCt CASE.}

In view of Corollary 3.3, the main obstacle towards a complete proof is in compact non-metrizable groups. Our main tool to solve this case will be the results of Grekas and Mercourakis in [28], where it is proved that every compact group $K$ may be sandwiched, from the measure and topological point of view, between two groups of the form $M \times H$ with $M$ non-discrete and metrizable.

Following [28], we say that two locally compact groups groups $G_{1}$ and $G_{2}$ with Haar measures $\lambda_{1}$ and $\lambda_{2}$, respectively, are Haar homeomorphic when there exists a homeomorphism $\psi: G_{1} \rightarrow G_{2}$ such that $A \subset G_{1}$ is $\lambda_{1}$-measurable if and only if $\psi(A)$ is $\lambda_{2}$-measurable and $\lambda_{1}(A)=\lambda_{2}(\psi(A))$. Note that in this situation, the map $f \mapsto f \circ \psi$ establishes a linear isometry between the group algebras $L^{\infty}\left(G_{1}\right)$ and $L^{\infty}\left(G_{2}\right)$ as well as between the $C^{*}$-algebras $\mathcal{C B}\left(G_{1}\right)$ and $\mathcal{C} \mathcal{B}\left(G_{2}\right)$, and so these spaces may be identified.

We start by recalling Grekas and Mercourakis results, which will help us to deal with non-metrizable compact groups. For our first Lemma we do not need the full force of the corresponding Theorems in [28]. Even if Lemma 5.1 in this form follows easily from [32, Corollary 9.25] and the structure of Abelian groups, we find it more convenient to lean on Theorem 1.1 of [28] which already contains these arguments.

Lemma 5.1. [28, Theorems 1.1(1) and 1.4(1)] Let $K$ be a non-trivial compact, connected group. There are two families of compact non-trivial connected metrizable groups $\left\{M_{i}: i \in I\right\}$ and $\left\{N_{i}: i \in I\right\}$ and two continuous and open surjective homomorphisms $\varphi_{1}$ and $\varphi_{2}$ as follows:

$$
\prod_{i} M_{i} \stackrel{\varphi_{2}}{\longrightarrow} K \stackrel{\varphi_{1}}{\longrightarrow} \prod_{i} N_{i}
$$

Lemma 5.2. [28, Theorem $1.1(2)]$ Let $K$ be a totally disconnected, compact group. There is a Haar homeomorphism $\phi: K \rightarrow \prod_{i} F_{i}$, where each $F_{i}$ is a finite group. 
We first observe the that the existence of self-isometries of $L^{\infty}(K)$ preserved by $\mathcal{C} \mathcal{B}(K)$ is preserved by Haar homeomorphism.

Lemma 5.3. Let $G$ and $H$ be Haar homeomorphic groups. Suppose that $\Psi_{H}: L^{\infty}(H) \rightarrow L^{\infty}(H)$ is a linear isometry that preserves $\mathcal{C} \mathcal{B}(H)$. Then $\Psi_{H}$ induces a linear isometry $\Psi_{G}: L^{\infty}(G) \rightarrow L^{\infty}(G)$ that preserves $\mathcal{C} \mathcal{B}(G)$.

Proof. If $j: H \rightarrow G$ denotes a Haar homeomorphism, the required isometry will be given by $\Psi_{G} \xi=\Psi_{H}(\xi \circ j) \circ j^{-1}$. Since $\phi \circ j \in \mathcal{C B}(H)$ for every $\phi \in \mathcal{C B}(G)$, the proof is straightforward.

Theorem 5.4. Let $K$ be a compact group. If $K$ is infinite, then there exists a linear isometric copy of $L^{\infty}(K)$ into $L^{\infty}(K)$ that is preserved by $\mathrm{eB}(K)$. The group algebra $L^{1}(K)$ is, in particular, extremely non-Arens regular.

Proof. If $K$ is metrizable, Corollary 3.3 with $K=M$ and $H=\{e\}$ yields the theorem.

Suppose now that $K$ is not metrizable. As a compact group, $K$ is Haar homeomorphic to $K_{0} \times\left(K / K_{0}\right)$, where $K_{0}$ denotes the connected component of $K$ (see [28, Theorem (B) and the remarks thereafter], see also [42, Theorem 8]). By Lemma 5.3, it is sufficient to prove the theorem for $K_{0} \times\left(K / K_{0}\right)$. Note that either $K_{0}$ or $K / K_{0}$ is not metrizable.

We suppose first that $K / K_{0}$ is metrizable. Then $K_{0}$ is non-metrizable. By Lemma 5.1 we have two collections of compact connected metrizable groups

$$
\left\{M_{i}: i \in I\right\} \quad \text { and } \quad\left\{N_{i}: i \in I\right\}
$$

and two continuous and open surjective homomorphisms $\varphi_{1}$ and $\varphi_{2}$

$$
\prod_{i} N_{i} \stackrel{\varphi_{2}}{\longrightarrow} K_{0} \stackrel{\varphi_{1}}{\longrightarrow} \prod_{i} M_{i} .
$$

We check that $K_{0} \times\left(K / K_{0}\right)$ satisfies the conditions of Lemma 4.2.

We start by fixing any $i_{0} \in I$. The groups $M_{i_{0}} \times\left(K / K_{0}\right)$ and $N_{i_{0}} \times\left(K / K_{0}\right)$ are then metrizable and non-discrete. Then let $J=I \backslash\left\{i_{0}\right\}$ and put

$$
\begin{aligned}
M & =M_{i_{0}} \times\left(K / K_{0}\right), \\
H & =\prod_{i \in J} M_{i}, \\
K_{0} \times K / K_{0} & \stackrel{\varphi}{\longrightarrow} \prod_{i \in I} M_{i} \times\left(K / K_{0}\right)=M \times H,
\end{aligned}
$$

where $\varphi$ is defined as $\varphi=\varphi_{1} \times \mathrm{id}_{K / K_{0}}$. Condition (i) of Lemma 4.2 is trivially fulfilled. As to Condition (ii), since $I$ is infinite and the groups in both collections are metrizable, we have

$$
|I|=w\left(\prod_{i \in I} N_{i}\right) \geq w\left(K_{0}\right) \geq w\left(\prod_{i \in I} M_{i}\right)=|I| .
$$

Since $K / K_{0}$ is metrizable, this gives $w\left(K_{0} \times K / K_{0}\right)=w\left(K_{0}\right)=|I|$. Therefore, by Theorem 3.1 of [27] (and Maharam's theorem, see, e.g., [22, Theorem 331I]), both spaces

$$
L^{\infty}(M \times H)=L^{\infty}\left(\prod_{i \in I} M_{i}\right) \quad \text { and } \quad L^{\infty}\left(K_{0} \times K / K_{0}\right)
$$


are linearly isometric to $L^{\infty}\left(\{0,1\}^{|I|}\right)$, whence the existence of the linear isometry

$$
\Psi_{1}: L^{\infty}\left(K_{0} \times K / K_{0}\right) \rightarrow L^{\infty}(M \times H)
$$

required in Condition (ii) of Lemma 4.2 .

Since the map $\varphi$ clearly satisfies Condition (iii) of Lemma 4.2, we can conclude that that there is a linear isometry of

$$
L^{\infty}\left(K_{0} \times K / K_{0}\right) \rightarrow L^{\infty}\left(K_{0} \times K / K_{0}\right)
$$

preserved by $\mathcal{C} \mathcal{B}\left(K_{0} \times K / K_{0}\right)$. The existence of a linear isometry of $L^{\infty}(K)$ into $L^{\infty}(K)$ preserved by $\mathcal{C} \mathcal{B}(K)$ follows from Lemma 5.3.

Assume now that $K / K_{0}$ is not metrizable. By Lemma 5.2 we have now an uncountable collection of finite groups $\left\{F_{i}: i \in I\right\}$ and a Haar homeomorphism

$$
\varphi: K / K_{0} \longrightarrow \prod_{i \in I} F_{i}
$$

Fix an infinite countable subset $J$ of $I$ and let

$$
M=\prod_{i \in J} F_{i} \quad \text { and } \quad H=K_{0} \times \prod_{i \in I \backslash J} F_{i} .
$$

Then $M$ is a compact, non-discrete and metrizable subgroup of $K$ and $\mathrm{K}$ is Haar homeomorphic to $M \times H$. Corollary 3.3 and Lemma 5.3 yield the desired claim.

\section{The final step. Putting things together}

After having dealt with metrizable groups and compact groups, we will now use a classic theorem of Davis, reminiscient of a basic structural result of Yamabe [52], to the effect that every locally compact group has an open subgroup Haar homeomorphic to the product $\mathbb{R}^{n} \times K$ with $K$ compact.

We first observe how extreme non-Arens regularity of a locally compact group can be lifted from open subgroups.

Lemma 6.1. Let $G$ be a locally compact group and $G_{0}$ be an open subgroup of $G$. Suppose that $T: L^{\infty}\left(G_{0}\right) \rightarrow L^{\infty}\left(G_{0}\right)$ is an isometry preserved by $\mathcal{C} \mathcal{B}\left(G_{0}\right)$. Then $T$ extends to an isometry $\Psi: L^{\infty}(G) \rightarrow L^{\infty}(G)$ that is preserved by $\mathcal{e} \mathcal{B}(G)$.

Proof. Let $\left\{x_{i}: i \in I\right\}$ be a system of representatives of the left cosets of $G_{0}$ in $G$ with $|I|=\left|G: G_{0}\right|$.

For any $\xi \in L^{\infty}(G)$ and each $i \in I$, define $\xi_{i} \in L^{\infty}\left(G_{0}\right)$ by $\xi_{i}(s)=\xi\left(x_{i} s\right)$. Since $A \subset G$ is locally null if and only if $A \cap x_{i} G_{0}$ is locally null for every $i \in I$ (see [20, Page 46]), the correspondence

$$
\begin{aligned}
S: L^{\infty}(G) & \longrightarrow \ell^{\infty}\left(I, L^{\infty}\left(G_{0}\right)\right) \\
\xi & \longmapsto\left(\xi_{i}\right)_{i \in I}
\end{aligned}
$$

is a well-defined linear isometry.

Now let $\ell^{\infty}(I, T): \ell^{\infty}\left(I, L^{\infty}\left(G_{0}\right)\right) \rightarrow \ell^{\infty}\left(I, L^{\infty}\left(G_{0}\right)\right)$ denote the natural extension of $T$ to $\ell^{\infty}\left(I, L^{\infty}\left(G_{0}\right)\right)$ given by

$$
\ell^{\infty}(I, T)\left(\left(\xi_{i}\right)_{i \in I}\right)=\left(T \xi_{i}\right)_{i \in I} \quad \text { for } \quad\left(\xi_{i}\right)_{i \in I} \in \ell^{\infty}\left(I, L^{\infty}\left(G_{0}\right)\right) .
$$


We then define

$$
\Psi=S^{-1} \circ \ell^{\infty}(I, T) \circ S: L^{\infty}(G) \rightarrow L^{\infty}(G) .
$$

If $\xi \in L^{\infty}(G)$ and $\phi \in \mathcal{C B}(G)$, observing that $\phi_{i} \in \mathcal{C} \mathcal{B}\left(G_{0}\right)$ for every $i \in I$, we have that

$$
\begin{aligned}
\|\Psi(\xi)-\phi\|_{L^{\infty}(G)} & =\left\|\left(\ell^{\infty}(I, T) \circ S\right)(\xi)-S(\phi)\right\|_{\ell^{\infty}\left(I, L^{\infty}\left(G_{0}\right)\right)} \\
& =\sup _{i \in I}\left\|T\left(\xi_{i}\right)-\phi_{i}\right\|_{L^{\infty}\left(G_{0}\right)} \\
& \geq \sup _{i \in I}\left\|\xi_{i}\right\|_{L^{\infty}\left(G_{0}\right)} \\
& =\|S(\xi)\|_{\ell_{\left(I, L^{\infty}\left(G_{0}\right)\right)}} \\
& =\|\xi\|_{L^{\infty}(G)} .
\end{aligned}
$$

This is the theorem of Davis mentioned before:

Lemma 6.2 (Davis, [12]). Every locally compact group $G$ contains an open subgroup $H$ which is Haar homeomorphic to $\mathbb{R}^{n} \times K$ where $K$ is a compact group.

Proof. This follows directly from the Theorem in [12] and its proof.

We reach now our aim. We start with Theorem B.

Theorem 6.3. Let $\mathcal{F}(G)$ be any closed subspace of $\mathcal{C} \mathcal{B}(G)$. Then there exists a linear isometric copy of $L^{\infty}(G)$ in the quotient space $L^{\infty}(G) / \mathcal{F}(G)$ for any non-discrete, locally compact group $G$.

Proof. By the previous lemma, $G$ contains an open subgroup $G_{0}$ which is Haar homeomorphic to $\mathbb{R}^{n} \times K$.

If $n \neq 0$, we apply Corollary 3.3 to $\mathbb{R}^{n} \times K$ (with $H=K$ ). Lemma 5.3 and Lemma 6.1 then prove the existence of a linear isometry of $L^{\infty}(G)$ into $L^{\infty}(G)$ that is preserved by $\mathcal{C} \mathcal{B}(G)$ and we obtain the desired isometry from $L^{\infty}(G)$ into $L^{\infty}(G) / \mathcal{F}(G)$ (via Lemma 2.2 ).

If $n=0$ we follow the same path replacing Corollary 3.3 by Theorem 5.4 .

Only the discrete case of Theorem A has been left apart. But this is already known, see [19, Corollary3.4] or [7, Theorem 4.5] for two different proofs. Using our recent approach in [16] (see Theorem 2.11 thereof), both proofs can be now simplified. For completeness, we include below the shortened proof. Recall that a subset $T$ of $G$ is a $\mathcal{W} \mathcal{A P}(G)$-interpolation set when for every $f \in \ell^{\infty}(T)$ there is $\tilde{f} \in \mathcal{W} \mathcal{A P}(G)$ with $\left.\tilde{f}\right|_{T}=f$, i.e., when any bounded function on $T$ extends to the group $G$ as a weakly almost periodic function.

Theorem 6.4. Let $G$ be an infinite discrete group. Then the group algebra $\ell^{1}(G)$ is extremely non-Arens regular. 
Proof. Let $\kappa=|G|$, and $\left\{G_{\alpha}\right\}_{\alpha<\kappa}$ be an increasing family of symmetric subsets of $G$ with $\left|G_{\alpha}\right| \leq \alpha$ for every $\alpha<\kappa$ and such that $G=\bigcup_{\alpha<\kappa} G_{\alpha}$. Collect by induction a set $X=\left\{t_{\alpha}: \alpha<\kappa\right\}$ such that

$$
G_{\alpha} t_{\alpha} G_{\alpha} \cap G_{\beta} t_{\beta} G_{\beta}=\varnothing \quad \text { for every } \quad \alpha \neq \beta<\kappa .
$$

This is possible since $\left|G_{\alpha}\left(\bigcup_{\beta<\alpha} G_{\beta} t_{\beta} G_{\beta}\right) G_{\alpha}\right| \leq \alpha<\kappa$. Partition $\kappa$ into $\kappa$-many subsets $\kappa=\bigcup_{\eta<\kappa} I_{\eta}$, each having cardinality $\left|I_{\eta}\right|=\kappa$. We claim that each set $T_{\eta}=\bigcup_{\alpha \in I_{\eta}} G_{\alpha} t_{\alpha} G_{\alpha}$ fails to be a $\mathcal{W} \mathcal{A P}(G)$-interpolation set. So, fix $\eta<\kappa$, put $I_{\eta}=A_{\eta} \cup B_{\eta}$ with $A_{\eta} \cap B_{\eta}=\varnothing$ and consider the function $f$ which is 1 on $\bigcup_{\alpha \in A_{\eta}} G_{\alpha} t_{\alpha} G_{\alpha}$ and 0 on $\bigcup_{\alpha \in B_{\eta}} G_{\alpha} t_{\alpha} G_{\alpha}$. Then for each fixed $\alpha$ and for $\beta$ sufficiently large, $t_{\alpha} \in G_{\beta}$ and so $t_{\alpha} t_{\beta} \in G_{\beta} t_{\beta}$. If $\beta \in B_{\eta}$, this implies that $f\left(t_{\alpha} t_{\beta}\right)=0$. On the other hand, for each fixed $\beta$ and for $\alpha$ sufficiently large $t_{\beta} \in G_{\alpha}$ and so $t_{\alpha} t_{\beta} \in t_{\alpha} G_{\alpha}$. If $\alpha \in A_{\eta}$, this implies that $f\left(t_{\alpha} t_{\beta}\right)=1$. Accordingly,

$$
\lim _{\alpha \in A_{\eta}} \lim _{\beta \in B_{\eta}} f\left(t_{\alpha} t_{\beta}\right)=0 \quad \text { while } \quad \lim _{\beta \in B_{\eta}} \lim _{\alpha \in A_{\eta}} f\left(t_{\alpha} t_{\beta}\right)=1 \text {. }
$$

This shows that $f$ cannot be extended to $G$ as a function in $\mathcal{W} \mathcal{A P}(G)$. By $\left[16\right.$, Theorem 2.11], there is then a linear isometry of $\ell^{\infty}(\kappa)$ into the quotient $\ell^{\infty}(G) / \mathcal{W} \mathcal{A P}(G)$. Therefore, $\ell^{1}(G)$ is extremely non-Arens regular.

And here is Theorem A.

Theorem 6.5. Let $G$ be an infinite locally compact group. Then $L^{1}(G)$ is extremely non-Arens regular.

Proof. If $G$ is non-discrete, this is an immediate consequence of Theorem 6.3. If $G$ is discrete, the claim was proved in [19] or [7].

Let $A(G)$ be the Fourier algebra, consisting of all functions $u \in C_{0}(G)$ of the form $f * \check{g}(x \in G)$, where $f, g \in L^{2}(G)$ and $\check{g}(x)=\overline{g\left(x^{-1}\right)}$. The norm in $A(G)$ is given by

$$
\|u\|=\inf \left\{\|f\|_{2}\|g\|_{2}: u=f * \check{g}, f, g \in L^{2}(G)\right\}
$$

(see Eymard [14]). In harmonic analysis, $A(G)$ is seen as the dual object of the group algebra $L^{1}(G)$. In fact when $G$ is abelian, the Fourier transform is an isometric algebra isomorphism from $L^{1}(\widehat{G})$ onto $A(G)$.

As already noted at the begining of the paper, the extreme non-Arens regularity of the Fourier algebra $A(G)$ was proved by Hu in [33] when $\chi(G) \geq$ $\kappa(G)$. The question of whether $A(G)$ is extremely non-Arens regular for any infinite locally compact group remains open. But when $G$ is Abelian, as an immediate consequence of Theorem 6.5 , we can omit the condition $\chi(G) \geq \kappa(G)$.

Corollary 6.6. If $G$ is an infinite locally compact Abelian group, then the Fourier algebra $A(G)$ is extremely non-Arens regular 
Acknowledgments. We would like to thank the referee for her/his very careful reading of the paper, the corrections and for the useful comments.

Parts of the article were written when the first named author was visiting Jaume I University in Castellón in December 2011 and May 2012. He would like to express his warm thanks for the kind hospitality and support. Subsequently, he was partially supported by Väisälä Foundation in 2012-2014. This support is gratefully acknowledged.

Research of the second named author supported by Ministerio de Economa y Competitividad (Spain) through project MTM2016-77143-P (AEI/FEDER, UE). This support is also gratefully acknowledged.

\section{REFERENCES}

[1] R. Arens, The adjoint of a bilinear operation, Proc. Amer. Math. Soc. 2, (1951), 839-848.

[2] R. Arens, Operations induced in function classes, Monatsh. Math. 55 (1951), 1-19.

[3] A. Arhangel'skii and M. Tkachenko, Topological groups and related structures, Atlantis Studies in Mathematics, vol. 1, Atlantis Press, Paris, 2008.

[4] J. F. Berglund, H. D. Junghenn and P. Milnes, Analysis on Semigroups: Function Spaces, Compactifications, Representations, Wiley, New York (1989).

[5] F. F. Bonsall and J. Duncan, Complete normed algebras, Springer-Verlag, Berlin, 1973.

[6] T. Budak, N. Issik and J. Pym, Minimal determinants of topological centres for some algebras associated with locally compact groups, Bull. Lond. Math. Soc. 43 (2011), no. 3, 495-506.

[7] A. Bouziad and M. Filali, On the size of quotients of function spaces on a topological group, Studia Math. 202 (2011), 243-259.

[8] C. Chou, Weakly almost periodic functions and Fourier-Stieltjes algebras of locally compact groups, Trans. Amer. Math. Soc., 274 (1982), no. 1, 141-157.

[9] P. Civin and B. Yood, The second conjugate space of a Banach algebra as an algebra, Pacific J. Math. 11 (1961), 847-870.

[10] H. G. Dales, Banach algebras and automatic continuity, London Math. Society Monographs, Volume 24, Clarendon Press, Oxford, 2000.

[11] H. G. Dales and, A. T.-M. Lau, The second duals of Beurling algebras, Mem. Amer. Math. Soc. 177 (2005).

[12] H. F. Davis. A note on Haar measure. Proc. Amer. Math. Soc., 6 (1955), 318-321.

[13] M. M. Day, Amenable semigroups, Illinois J. Math. 1 (1957), 509-544.

[14] P. Eymard, L'algèbre de Fourier d'un groupe localement compact, Bull. Soc. Math. France 92 (1964), 181-236.

[15] M. Filali and J. Galindo, Approximable $\mathcal{W} \mathcal{A P}$ - and LUC-interpolation sets, Adv. Math. 233 (2013), 87-114.

[16] M. Filali and J. Galindo, Interpolation sets and the size of quotients of function spaces on a locally compact group, Trans. Amer. Math. Soc. 369 (2017), 575-603.

[17] M. Filali and P. Salmi, Slowly oscillating functions in semigroup compactification and convolution algebras, Journal of Functional Analysis 250 no. 1 (2007) 144-166.

[18] M. Filali and A. Singh, Recent developments on Arens regularity and ideal structure of the second dual of a group algebra and some related topological algebras, Est. Math. Soc., Tartu (2001) 95-124.

[19] M. Filali and T. Vedenjuoksu, Extreme non-Arens regularity of semigroup algebras, Topology Proc. 33 (2009), 185-196.

[20] G. B. Folland, A Course in Abstract Harmonic Analysis, CRC Press, Boca Raton, 1995.

[21] G. B. Folland, Real Analysis, John Wiley, New York, 1984.

[22] D. H. Fremlin. Measure theory. Vol. 3. Measure algebras.. Torres Fremlin, Colchester, 2004 . 
[23] C. K. Fong and M. Neufang, On the quotient space $U C(G) / W A P(G)$ and extreme non Arens regularity of $L^{1}(G)$, prep rint (2006).

[24] B. Forrest, Arens regularity and discrete groups, Pacific J. Math. 151 (1991), 217-227.

[25] E. E. Granirer, Day points for quotients of the Fourier algebra $A(G)$, extreme nonergodicity of their duals and extreme non-Arens regularity, Illinois J. Math. 40 no. 3 (1996) 402-419.

[26] M. Grosser and V. Losert, The norm strict bidual of a Banach algebra and the dual of $C_{u}(G)$, Manuscripta Math. 45 (1984), 127-146.

[27] S. Grekas, Isomorphic measures on compact groups, Math. Proc. Cambridge Philos. Soc. 112 (1992), 349-360.

[28] S. Grekas and S. Mercourakis. On the measure-theoretic structure of compact groups, Trans. Amer. Math. Soc. 350 (1998), no. 7, 2779-2796.

[29] A. Grothendieck, Critères de compacité dans les espaces fonctionnels généraux, Amer. J. Math. 74 (1952), 168-186.

[30] S. L. Gulick, Commutativity and ideals in the biduals of topological algebras, Pacific J. Math. 18 (1966), 121-137.

[31] E. Hewitt and K. A. Ross, Abstract harmonic analysis. Vol. I: Structure of topological groups, integration theory, group representations, Academic Press Inc., Publishers, New York (1963).

[32] K. H. Hofmann and S. A. Morris, The structure of compact groups. A primer for the student-a handbook for the expert., 2nd edition. Walter de Gruyter \& Co., Berlin, 2006.

[33] Z. Hu. Extreme non-Arens regularity of quotients of the Fourier algebra $A(G)$. Colloq. Math. 72 (1997), no. 2, 237-249.

[34] Z. Hu and M. Neufang, Decomposability of von Neumann Algebras and the Mazur Property of Higher Level, Canad. J. Math. 58 (2006) 768-795.

[35] Z. Hu and M. Neufang Distinguishing properties of Arens irregularity, Proc. Amer. Math. Soc. 137 (2009), no. 5, 1753-1761.

[36] Z. $\mathrm{Hu}$, On the set of topologically invariant means on the von Neumann algebra $V N(G)$, Illinois J. Math. 39 (1995), 463-490.

[37] N. Issik.; J. Pym and A. Ülger, The second dual of the group algebra of a compact group, J. London Math. Soc. (2) 35 (1987), no. 1, 135-148.

[38] A. T. M. Lau and V. Losert, On the second conjugate of $L^{1}(G)$ of a locally compact group, J. London Math. Soc. 37 (1988), 464-470.

[39] A. T.-M. Lau and J. C. S. Wong, Weakly almost periodic elements in $L^{\infty}(G)$ of a locally compact group, Proc. Amer. Math. Soc. 107 (1989), 1031-1036

[40] V. Losert, Talk in Abstract Harmonic Analysis Conference, Istanbul (2006).

[41] V. Losert, The centre of the bidual of Fourier algebras (discrete groups), Bull. London Math. Soc. 48 (2016), 968-976.

[42] P. S. Mostert, Sections in principal fibre spaces, Duke Math. J. 23 (1956), 57-71.

[43] M. Neufang, A unified approach to the topological centre problem for certain Banach algebras arising in abstract harmonic analysis, Arch. Math. (Basel) 82 (2004), no. 2, 164-171.

[44] T. W. Palmer, Banach algebras and the general theory of *-algebras, Volume 1, Algebras and Banach algebras, Cambridge University Press, 1994.

[45] J. S. Pym, The convolution of functionals on spaces of bounded functions, Proc. London Math. Soc. 15 (1965), 84-104.

[46] H. Reiter and J. D. Stegeman. Classical Harmonic Analysis and Locally Compact Groups., 2nd ed. Clarendon Press, Oxford, 2000.

[47] H. L. Royden, Real analysis, The Macmillan Co., New York; Collier-Macmillan Ltd., London 1963.

[48] H. P. Rosenthal, On injective Banach spaces and the spaces $L^{\infty}(\mu)$ for finite measures $\mu$, Acta Math. 124 (1970), 205-248.

[49] S. Sherman, The second adjoint of a $C^{*}$-algebra, Proc. International Congress Mathematicians, Cambridge, Mass., Volume 1, (1950), 470.

[50] Z. Takeda, Conjugate spaces of operator algebras, Proc. Japan Academy, 30 (1954), 90-95. 
[51] A.Ülger, Continuity of weakly almost periodic functionals on $L^{1}(G)$, Quart. J. Math. Oxford Ser. (2) 37 (1986) 495-497.

[52] H. Yamabe, A generalization of a theorem of Gleason, Ann. of Math. vol. 58 (1953) 351-365.

[53] N. J. Young, Separate continuity and multilinear operations, Proc. London Math. Soc. 26 (1973), 289-319.

[54] N. J. Young, The irregularity of multiplication in group algebras, Quart J. Math. Oxford Ser. 24 (1973), 59-62.

Mahmoud Filali, Department of Mathematical Sciences, University of Oulu, OUlu, Finland

E-MAIL: mfilali@cc.oulu.fi

Jorge Galindo, Instituto Universitario de Matemáticas y Aplicaciones (IMAC), Universidad Jaume I, E-12071, Castellón, Spain.

E-MAIL: jgalindo@mat.uji.es 